\title{
A Survey on Time-aware Business Process Modeling
}

\author{
Saoussen Cheikhrouhou ${ }^{1}$, Slim Kallel ${ }^{1}$, Nawal Guermouche ${ }^{2,3}$ and Mohamed Jmaiel ${ }^{1}$ \\ ${ }^{1}$ ReDCAD Laboratory, National Engineering School of Sfax, University of Sfax B.P. 1173, 3038 Sfax, Tunisia \\ ${ }^{2}$ CNRS-LAAS, 7 avenue du colonel Roche, F-31400 Toulouse, France \\ ${ }^{3}$ Univ de Toulouse, INSA, LAAS, F-31400 Toulouse, France \\ saoussen.cheikhrouhou@redcad.org,Slim.kallel@fsegs.rnu.tn, Nawal.Guermouche@laas.fr, \\ mohamed.jmaiel@enis.rnu.tn
}

\begin{abstract}
Keywords:
Temporal constraints and dependencies : Business Process Modeling (BPM) : Workflow : Web service composition : Inter-Organisational Business Process (IOBP).
\end{abstract}

Abstract:

One key perspective when dealing with Business Process Management (BPM) is time. All business experts agree upon the fact that time is a key resource for each business process within an organisation. Indeed, time managing is an effective cost and error reduction strategy and thus ensures profit maximization for the organisation. As a result, business managers, researchers, and academicians in management are striving to have full-support of temporal aspects in business process management suites. Consequently, modeling and managing temporal requirements in the business process field is becoming a topic of intensive research. This paper presents a survey of the existing approaches to specifying and verifying the temporal perspective in business processes.

\section{INTRODUCTION}

Nowadays, business is migrating from Business-to-Comsumer (B2C) applications to Business-to-Business (B2B) ones in order to deal with the ever increasing economic pressure and to enhance the overall competitiveness. When addressing the issue of $\mathrm{B} 2 \mathrm{~B}$, one organisation may collaborate with many others with complementary skills to form an Inter-Organisational Business Process (IOBP). The emergence of the IOBP field gave already a major contribution to different sectors such as aeronautic domain, in which more than $50 \%$ of the supply chain is subcontracted. Furthermore, the aeronautic sector's strategy is migrating from one-tier subcontractor to an important number of subcontractors with a ditributed control over the different sub-contracting processes. Satisfying time constaints such as time deadlines is vital for the processes of the aviation in- dustry, since the violation of such constraints may lead to critical situations and could even threaten the aviation safety. Obviously, it is not straightforward to adress the temporal requirements in IOBPs.

Different specification methods and verification techniques and tools have been developed to deal with such setting (Bettini et al., 2002; Kazhamiakin et al., 2006a; Guermouche, 2010; Watahiki et al., 2011). Nevertheless, the temporal resource management in business processes, especially in huge and collaborative processes as used in the aviation industry, is still a challenging research task.

This paper surveys the current state of the art in specifying and verifying the temporal perspective in business processes. But the main focus of this paper is the specification step. Few research attempts however have been made to carry overviews on the business process field (see, for instance (Wang, 2004; Dustdar and Schreiner, 2005)). Nevertheless, 
these overviews are not as focused as the one presented here since they do not elect time as a first time citizen in the business process modeling (BPM) phase.

This paper is structured as follows. Section 1 gives an overview on the existing temporal constraints specification and verification methods in the business process field, followed by a rich evaluation and discussion in section 2 . The last section concludes and highlights the emerging research challenges to address in the field of business process time management.

\section{OVERVIEW ON THE EXISTING TEMPORAL CONSTRAINTS SPECIFICATION METHODS}

As preliminary step of this work, we give a classification of the existing temporal constraints models. Mainly, the studied approaches are collected from three research areas: workflows, Web service composition, and inter-organisational domain. These research areas can be generalized and seen from a business process field perspective.

\subsection{Temporal constraints in the workflow research area}

The major contribution of TimeBPMN (Gagné and Trudel, 2009), is the extension of Business Process Modeling Notation BPMN (OMG, 2008) with a large set of required temporalities. This extension deals with various temporal constraints and dependencies between business process activities. However, this extension does not permit to model temporal constraints relating to the duration of business process activities (eg. A given activity lasts $\mathrm{x}$ time units and $\mathrm{x}$ may be defined as a time interval). In addition, Time-BPMN is limited to the specification phase since no verification mechanism of temporal constraints conflicts is provided.

The work presented in (Watahiki et al.,
2011) proposes a formal specification of BPMN (OMG, 2008) with timed automata. First, the authors extend BPMN to handle temporal constraints (i.e., the minimum and maximum execution time of a task), resource constraints, and concurrency constraints (i.e., the number of instances executable in parallel). Second, they provide an automatic mapping of the extended BPMN onto timed automata. Computation tree logic (CTL) formulas are used to specify the different properties to be verified by the UPAAL model checker. This approach aims at verifying some features, such as deadlocks and bottlenecks. The scope of this paper is limited to a small subset of BPMN elements. Additionnally, this BPMN extension permits to specify temporal constraints related to only one activity within the business process model.

Huai et al. (Huai et al., 2010) present a method for verifying BPMN (OMG, 2008) models based on time Petri nets. The proposed method supports the analysis of model structure (dead task, deadlock and infinite loops) and tests the time conflicts of the model. First, the authors translate the BPMN model to time Petri nets. Second, they construct the reachability graph of the Petri nets in order to verify the model structure. Furthermore, they exhibit the time choreography verification algorithm to verify time conflicts. The proposed algorithm proceeds by the accumulation of clock constraints of terminated activities and assignes them to the corresponding activated activities within the tested path. This work presents the advantage that clock constraints are propagated to tasks and message flows, which makes explicit the implicit timed conflicts due to service interaction. The first limitation is related to the lack of temporal dependencies between multiple activities of the business process, which makes the proposed time choreography verification algorithm very limited.

The major contribution of the approach cited in (Du et al., 2011) is that it can dynamically check the temporal violations of multiple concurrent workflow processes with resource constraints. First, the authors construct the sprouting graph models of the time workflow nets (TWF-nets) (Ling and Schmidt, 2000) 
for multiple workflow processes. Second, they update the sprouting graph at different checking points and check the temporal constraints. Finally, and most importantly, the violation paths and solutions (by modifying the duration of some activities) are given. Moreover, they use the UPAAL model checker to verify the correctness of their approach. This work verifies only temporal constraints of the form: an activity $a_{j}$ should end its execution no later than $\mathrm{x}$ time units after the activity $a_{i}$ starts. The complexity of the construction of the sprouting graph becomes high when the number of resource constraints increases. This is identified as the major weakness of this approach.

The particularity of the approach of Bettini et al. (Bettini et al., 2002) is that it merges several research directions on temporal workflow models and on temporal constraint networks. Regarding the modeling of temporal constraints, Temporal Constraint with Granularity TCG graph is used for this purpose. Additionally, this paper provides temporal constraints reasoning and management tool offering the following services: First, it checks the consistency of complex temporal requirements. Second, it monitors workflow activities and predicts their starting and ending time. Finally it provides the enactment service with useful temporal information for activity scheduling. Hence, the concept of Free schedules is a relevant contribution of this paper. A schedule is free when it is possible to statically fix the start times of all tasks of the workflow without constraining their durations while satisfying all the other temporal constraints.

Time modeling and management in the clinical workflow domain has been widely investigated by Combi et al. (Combi et al., 2007; Combi and Posenato, 2009). Among the proposed temporal constructs, we can notice: the duration (the activity duration) and delays (the edge duration), the relative constraints, the absolute constraints, and the periodic constraints. To this regard, the authors developed a tool named Temporal Workflow Analyzer (TWA) to support workflow modeling at workflow design time. Furthermore, in (Combi and Posenato, 2009), the authors introduced and discussed the concept of controllability checking which refers to the capability of executing a workflow for any possible duration of tasks.

Wong et al. have described in (Wong and Gibbons, 2009) a relative-timed semantic model for BPMN in the language of Communicating Sequential Processes (CSP). This model helps to introduce the notion of relative time in the form of delays and durations. The model checker FDR is accordingly used to verify the time compatibility issue in business collaborations.

\subsection{Temporal constraints in web service composition research field}

This subsection covers the basics of temporal constraints specification and verification approaches in the web service composition research field.

The authors in (Kazhamiakin et al., 2006a) address the problem of qualitative and quantitative analysis of timing aspects of web service compositions. To capture the timing aspects of BPEL4WS processes, the Web Service Timed State Transition Systems (WSTTS) formalism is previously introduced in (Kazhamiakin et al., 2006b). For the verification, they use the NuSMV model checker. The authors verify the composition against a large set of temporal properties such as deadlock and the termination of the procedure within a given delay. Furthermore, the approach aims at calculating the maximal and minimal duration time of the process. This work has the advantage not only to check whether a certain time-related requirement is satisfied, but also to compute extreme time bounds that satisfy such requirement. Nevertheless, considering only timing aspects of BPEL4WS processes limits the mentioned approach to the service oriented research field. Verifying the timing requirements on the model (exp. BPMN) results in a generalized approach applicable to a service oriented implementation as well as to other possible implementations.

The approach proposed in (Kallel et al., 2009) covers the specification of temporal con- 
straints for the web service domain using a new proposed language, XTUS-Automata. In the specification phase, this work presents temporal specification patterns (i.e. patterns for duration properties, pattern for temporal properties over cardinalities, and pattern for absolute time properties). This work combines timed automata (TA) and extended time unit system (XTUS) to allow specifying temporal properties involving relative time as well as absolute time. Furthermore, this work conducts a formal verification of deadlock using the model checker UPAAL. Finally, it presents an aspect-based monitoring mechanism, in which formally specified temporal constraints are translated automatically to modular aspect code in the aspect-oriented workflow language AO4BPEL. It is worth noting that this paper offers interesting specification patterns by which we can cover a large set of real world workflow temporal constraints. Nevertheless, this work is unable to verify the existence of temporal constraint conflicts.

In (Guermouche, 2010), the author uses temporal properties in order to analyze the compatibility in web service composition. A formal model abstracting messages, data, data constraints as well as temporal constraints, based on timed automata is proposed. A compatibility analysis of the web service choreography is conducted. The UPAAL model checker was used to detect some structural problems due to temporal conflicts. So far in this approach, the focus has been on the construction of a correct web service composition. For this end, a mediator is generated, whenever it is possible, to overcome the web service collaboration incompatibility issues. The clock ordering process is used to verify deadlock freeness due to time constraints conflicts. Nevertheless, the scope of this paper is limited to the verification of time constraints only caused by message interaction between services of the process.

In (Guermouche and Zilio, 2012), the authors propose a framework to check temporal requirements on choreographies. This is achieved by the verification of the composed annotated BPEL processes. This work enables efficiently to specify time constraints such as the estimated execution time of ac- tivities and the temporal delay between two activities or messages. Furthermore, complex temporal requirements could be expressed. For instance, the absence pattern with delay and the response pattern with delay. The timed business processes are automatically translated into the formal modeling language, Fiacre (Berthomieu et al., 2008). The TIme petri Net Analyzer (TINA) (Berthomieu et al., 2004) model checker tool is used for complex real-time requirements automatic checking. This work has the advantage of supporting synchronous as well as asynchronous services. This work has attempted to provide a modeling environment inspired from BPMN to visually specify the temporal requirements whereas no translation mechanism from the graphical specification to the used formal language is provided.

Benatallah et al. have widely invested in checking compatibility and replaceability analysis in timed ptotocols of web services (Benatallah et al., 2004; Benatallah et al., 2005). The approach detailed in (Benatallah et al., 2005) models business protocols as deterministic finite state machines. The scope of this work is limited to synchronous services and temporal requirements can only be associted to messages inside the same service.

\subsection{Temporal constraints in the inter-organisational research field}

This subsection is dedicated to explaining research attempts to specify and verify temporal constraints in inter-organisational business processes, crossing the organizational boundaries.

Eder et al. (Eder and Tahamtan, 2008b) focuses on checking temporal consistency in interorganizational workflows. In this context each organization contributes to the interorganizational workflow through its process view. Process views are a prevalent modeling approach for interorganizational workflows. They include a subset of the activities of the organisational private workflow needed for communication. Indeed, it allows to or- 
ganisations to well interact with others while preserving their organisational privacy. The proposed approach checks if the interorganizational workflow is temporally consistent by checking if its participating views are temporally consistent. For that, the authors use the concept of temporal plans and use timed activity graphs as the basic modeling language. It is clear that assuming that the different activities of the workflow have a deterministic duration is quite restrictive. Additionnally, this approach enables to specify only deadline constraints between the corresponding activities of the process(i.e. activities that communicate with each other). By checking only the corresponding activities of the different views, the authors implicitly suppose that the temporal constraints are synchronized based on message synchronization which is not always true because the views may not start executing at the same time and thus it is insufficient to compare only the intervals of the corresponding activities. In addition, the authors do not mention any other issue for full consistency like messaging conformance, data flow conformance or structural conformance.

Time conformance has been studied by Eder and Tahamtan in (Eder and Tahamtan, 2008a). It consists in checking whether a timed orchestration satisfies a timed choreography by generating temporal execution plans. The algorithm calculating the timed graphs and checking temporal conformance is detailed in (Eder and Tahamtan, 2008a). The conformance condition verifies that for each activity the sum of its earliest possible start and its duration must be less or equal to its latest allowed end for both best and worst cases. The durations of activities are presented by deterministic values which limits the scope of this work. The authors do not consider temporal constraints crossing the boundary of an activity or event-related temporal constraints.

In the context of Inter-Organizational Workflows, the approach detailed in (Makni et al., 2010) deals with the deadline constraints conformance verification, and thus without exposing the private processes of the involved partners. The authors demonstrate how missing deadlines while delivering the re- quired services may cause a global failure execution, even if the business behavior complementarity of the involved services is ensured. Based on the CoopFlow approach and using Time Petri nets theory, the authors propose a method for modeling and advertising temporal requirements for cooperative activities on the abstracted version of business processes by using observers. In fact, they prove that a deadline local verification process executed by a partner can lead to a deadline conformance in the resulting interconnected workflow. However, no method is proposed for the deadline local verification process. Additionally, this work permits only to add deadline constraints between cooperative activities (wich are visible by the cooperation candidates).

The authors in (Makni et al., 2011) discuss the application of Inter-Organizational Workflows (IOW) for automating processes in the collaborative context. A case study of emergency healthcare is presented in order to show the feasibility of the proposed temporal extension of the CoopFlow approach detailed in (Makni et al., 2010). This paper presents a proof of concept for automating the temporal conformance process in CoopFlow. The author noticed the use of TINA and Little Parametric Tool (LPT) tools (Godary, 2008) for verification purposes. Nevertheless, no more details are provided. Throughout this paper, the authors focused on mentioning negotiation aspects of temporal constraints in the presented case study but no negotiation strategy is detailed.

\section{EVALUATION AND DISCUSSION}

Throughout this survey paper, we provided a representative overview of the major efforts of time management in the business process field. The evaluation results are presented in table 1. Space limitations prevent presenting all the approaches discussed above, so we have omitted some of them being only focusing on a very limited scope of time management.

Table 1 highlights the different character- 
istics of each approach according to the following criteria:

\section{How temporal requirements are modeled within the approach?}

Eg. the standard BPMN, the TWF-nets, etc.

2. What properties against which the business process is verified?

Eg. the structural properties (i.e. the analysis of dead tasks, bottlenecks, deadlocks and loops), the time conflicts of the model, the user-defined temporal constraints (exp. the deadline constraints and the absence constraint), etc.

\section{How this verification is proceeded?}

Eg. model checker tools, algorithms, etc.

Based on the above observations, we identified that most of the already studied constraints include the temporal perspective. The temporal constraints are usually correlated with other constraints such as data (Guermouche, 2010) and resource constraints (Watahiki et al., 2011; Du et al., 2011).

Consequently, there are been several attempts to model the different constraints in the business process diagram itself using the defacto industrial standard for business process modeling, BPMN (Gagné and Trudel, 2009; Guermouche and Zilio, 2012). The use of a graph-based modeling approach of business processes as BPMN, is a competitive advantage. Indedeed, the visual appeal of the graph-based modeling approaches makes them useful for all kinds of workflow designers since no technical background is required. Similarly, other research efforts (Makni et al., 2010; Makni et al., 2011; Kallel et al., 2009; Guermouche, 2010; Du et al., 2011; Huai et al., 2010) opted for formal specification languages with modeling capabilities such as Petri nets and Timed Automata. Whereas, other approaches like (Wong and Gibbons, 2009) opted for CSP as a process algebra language, which lacks for graphical support. The approach followed by (Pesic et al., 2007) is somewhat different from the others since it models the constraints apart from the business process model (eg. by LTL formulas).
When considering the business process model itself, there are works specifying different constraints for one business process. Others take into account the cooperation between more than one business process both in the web service composition field and in the interorganizational business process field. When addressing the issue of IOBP, it is inevitable to reason about the migration of the different constraints between the private and the public workflows. There are several ideas for further research especially in the IOBP field. We can, first focus on elaborating a generic modeling approach which supports different constraint modeling such as temporal constraints and other associated constraints, namely, resource and data constraints. When dealing with temporal constraints, we remarked the lack of absolute time constraints in the majority of the works. In addition to that, just one work has modeled time points obtained from the execution phase (Kallel et al., 2009). We propose to deal with sequence, choice and concurrency structures and thus adopting best and worst cases of execution.

We now turn our attention to the temporal requirements verification problem. Temporal verification mechanisms are of paramount importance since they enable to detect, early on, possible temporal conflicts and to react to them effectively. In this context, although many efforts confound the time conflict verification of the model (i.e. the violation of some temporal requirements) with the structure verification (i.e. the analysis of dead tasks, bottlenecks, deadlocks and loops) (Kallel et al., 2009; Watahiki et al., 2011), there are some works which have tried to differentiate the two verification processes (Guermouche, 2010; Huai et al., 2010; Du et al., 2011). 
Table 1: Evaluation- How existing research approaches model and verify the temporal constraints (TC)

\begin{tabular}{|c|c|c|c|}
\hline $\begin{array}{c}\text { Approaches } \\
{[\mathrm{REF}]}\end{array}$ & $\begin{array}{c}\text { How TC } \\
\text { are modeled? }\end{array}$ & $\begin{array}{l}\text { What properties } \\
\text { are verified? }\end{array}$ & $\begin{array}{l}\text { How this verification } \\
\text { is proceeded? }\end{array}$ \\
\hline (Gagné and Trudel, 2009) & BPMN & & \\
\hline (Watahiki et al., 2011) & $\begin{array}{c}\text { BPMN } \\
+ \\
\text { Timed Automata } \\
\end{array}$ & $\begin{array}{c}\text { bottlenecks } \\
+ \\
\text { deadlocks } \\
\end{array}$ & UPAAL model checker \\
\hline (Huai et al., 2010) & Time Petri nets & \begin{tabular}{|c|} 
dead task + deadlock + infinite loops \\
the time conflicts of the model
\end{tabular} & $\begin{array}{l}\text { the reachability graph of the Petri nets } \\
\text { the time choreography verification algorithm }\end{array}$ \\
\hline (Du et al., 2011) & $\begin{array}{l}\text { Time workflow nets } \\
\text { (TWF-net) }\end{array}$ & $\begin{array}{c}\text { duration between two activitie } \\
\text { less than s time units }\end{array}$ & $\begin{array}{c}\text { Algorithms } \\
+ \\
\text { UPAAL model checker }\end{array}$ \\
\hline (Kazhamiakin et al., 2006a) & $\begin{array}{c}\text { Web Service Timed } \\
\text { State Transition Systems (WSTTS) }\end{array}$ & $\begin{array}{c}\text { deadlock } \\
+ \\
\text { maximal and minimal } \\
\text { duration of the process }\end{array}$ & model checker NuSMV \\
\hline (Kallel et al., 2009) & XTUS-Automata & deadlock & UPAALmodel checker \\
\hline \multirow[t]{2}{*}{ (Guermouche, 2010) } & \multirow[t]{2}{*}{ Timed Automata } & $\begin{array}{l}\text { compatibility analysis of the web } \\
\text { service choreography }\end{array}$ & Algorithms \\
\hline & & deadlock & UPAAL model checker \\
\hline $\begin{array}{l}\text { (Eder and Tahamtan, 2008b) } \\
\text { (Eder and Tahamtan, 2008a) }\end{array}$ & Timed activity graphs & $\begin{array}{c}\text { Temporal consistency } \\
+ \\
\text { Time conformance }\end{array}$ & Algorithms \\
\hline $\begin{array}{l}\text { (Makni et al., 2010) } \\
\text { (Makni et al., 2011) }\end{array}$ & Time Petri nets & Time conformance & TINA model checker and LPT \\
\hline (Guermouche and Zilio, 2012) & $\begin{array}{l}\text { A modeling environment } \\
\text { inspired from BPMN + } \\
\text { The Fiacre specification }\end{array}$ & $\begin{array}{l}\text { the absence constraint with delay } \\
\text { the response constraint with delay }\end{array}$ & TINA model checker \\
\hline (Bettini et al., 2002) & $\begin{array}{c}\text { Temporal Constraint with Granularity } \\
\text { (TCG) graph }\end{array}$ & the consistency & Algorithms \\
\hline $\begin{array}{c}\text { (Combi et al., 2007) } \\
\text { (Combi and Posenato, 2009) }\end{array}$ & $\begin{array}{c}\text { A proposed conceptual } \\
\text { workflow model }\end{array}$ & The controllability checking & Algorithms \\
\hline (Wong and Gibbons, 2009) & $\begin{array}{l}\text { Communicating Sequential } \\
\text { Processes (CSP) }\end{array}$ & Time compatibility & FDR model checker \\
\hline
\end{tabular}


To cope with the time conflict verification, there are some works which have neglected the intra-activity temporal requirements (eg. the duration of the modeled activities) (Guermouche, 2010). On the other hand, there are some approaches which have neglected the inter-activity temporal dependencies (Watahiki et al., 2011; Huai et al., 2010). Besides, the approach detailed in (Du et al., 2011) has tried to include the two different temporal requirements.

Additionally, there are some efforts concentrating on verifying other issues such as time conformance (Eder and Tahamtan, 2008a; Makni et al., 2010), the absence constraint (Guermouche and Zilio, 2012), and controllability (Combi and Posenato, 2009).

Once the verification process is conducted and a possible violation is detected, only few approaches (Du et al., 2011; Guermouche, 2010) tried to detect erroneous paths and to propose solutions. So far, the approach detailed in (Du et al., 2011) has proposed the modification of the duration of some activities as solution to the temporal violation in concurrent workflow processes with resource constraints. Additionnally, the author in (Guermouche, 2010) has considered the use of mediators when dealing with the compatibility analysis of the web service choreography. The idea of mediators proposed in (Guermouche, 2010) sounds very promising since it has succeeded in resolving a large set of temporal violations in an efficient manner.

Typically, researchers in the field of time management in the business process field are invited to widen the set of possible solutions to temporal constraints violations. From the research directions that have to be considered, we can notice the modification of the allocation policy of the shared resources and the change of the overall business process structure arriving at the substitution of some activities.

Finally, we can proceed by monitoring or enforcing of the different constraints in the execution phase. Another line of research is to study constraint-based business process models which offer design decisions at the execution time and enable different process variants.

\section{RESEARCH \\ CHALLENGES AND CONCLUSION}

Business managers, researchers, and academicians in management are striving to have full-support of temporal aspects in current business process management suites. Obviously, modeling and managing temporal requirements has long been a topic of intensive researches. Hence, with the help of the critical and comprehensive analysis presented within this survey paper, we pointed out that this emerging research field still face a multitude of challenges. The succeeding listing illustrates the major challenges to be addressed to substantially enhance the time management in the business process management field.

- Proposing a business process model supporting different temporal requirements : to enable the specification of temporal constraints related to one activity as well to Ad-Hoc sub processes and concurrent business processes sharing resources and exchanging messages.

- Improving the existing process view generation methods in order to define the mapping of a large set of temporal requirements from private to public process models.

- Defining a mapping mechanism from the business process model to a suitable formal language for future verification purposes. And if necessary, proposing a new formal language to well support the specification of all the temporal requirements.

- Suggesting efficient verification approaches to diagnose potential temporal violations of the process model early enough. In this context, it is beneficial to verify the business process against several issues such as structural properties (i.e. the analysis of dead tasks, bottlenecks, deadlocks and loops), time conflicts of the model, user-defined temporal constraints (exp. the deadline constraints and the absence constraint), time conformance of the IOBP (similarly the compatibility analysis in the web service field) as well as the controllability checking. 
- Defining violation identification mechanisms and proposing relevant primitives to resolve violations.

To summarize, in this paper we have analyzed and compared existing approaches for modeling and verifying time-related properties on business processes. Based on this analysis and evaluation, we have pointed out the challenges which sets foundations for full temporal support in business process modeling area. We are convinced that finding solutions to these challenges will significantly improve the interorganisational business process temporal support, helps to achieve process automatisation and thus helps the organisation to get advantage over competitors and to maximize its revenue.

\section{REFERENCES}

(2008). Object Management Group (OMG), Business Process Modeling Notation (BPMN), Version 1.1.

Benatallah, B., Casati, F., Ponge, J., and Toumani, F. (2005). On Temporal Abstractions of Web Service Protocols. In Proceedings of the 17th Conference on Advanced Information Systems Engineering (CAiSE), volume 161 of CEUR Workshop Proceedings, pages 13-17. CEURWS.org.

Benatallah, B., Casati, F., and Toumani, F. (2004). Analysis and Management of Web Service Protocols. In Proceedings of the 23rd International Conference on Conceptual Modeling, volume 3288 of LNCS, pages 524-541. Springer.

Berthomieu, B., Bodeveix, J.-P., Farail, P., Filali, M., Garavel, H., Gaufillet, P., Lang, F., and Ve rnadat, F. (2008). Fiacre: an Intermediate Language for Model Verification in the Topcased Environment. In Proceedings of the 4 th European Congress in EMBEDDED REAL TIME SOFTWARE, Toulouse, France.

Berthomieu, B., Ribet, P.-O., and Vernadat, F. (2004). The tool tina âA $\breve{S}$ construction of abstract state spaces for petri nets and time petri nets. International Journal of Production Research, 42(14):2741-2756.

Bettini, C., Wang, X. S., and Jajodia, S. (2002). Temporal Reasoning in Workflow Systems. Distributed and Parallel Databases, 11(3):269-306.

Combi, C., Gozzi, M., Juárez, J. M., Oliboni, B., and Pozzi, G. (2007). Conceptual Modeling of Temporal Clinical Workflows. In Proceedings of the 14th International Symposium on Temporal Representation and Reasoning (TIME), pages 70-81. IEEE Computer Society.

Combi, C. and Posenato, R. (2009). Controllability in Temporal Conceptual Workflow Schemata. In Proceedings of the rth International Conference on Business Process Management (BPM), volume 5701 of $L N C S$, pages 64-79. Springer.

Du, Y., Xiong, P., Fan, Y., and Li, X. (2011). Dynamic Checking and Solution to Temporal Violations in Concurrent Workflow Processes. IEEE Transactions on Systems, Man, and Cybernetics, Part A, 41(6):1166-1181.

Dustdar, S. and Schreiner, W. (2005). A survey on web services composition. IJWGS, 1(1):1-30.

Eder, J. and Tahamtan, A. (2008a). Temporal Conformance of Federated Choreographies. In Bhowmick, S. S., Küng, J., and Wagner, R., editors, Proceedings of the 19th International Conference on Database and Expert Systems Applications (DEXA), volume 5181 of $L N C S$, pages 668-675. Springer.

Eder, J. and Tahamtan, A. (2008b). Temporal Consistency of View Based Interorganizational Workflows. In Kaschek, R., Kop, C., Steinberger, C., and Fliedl, G., editors, Proceedings of the 2nd International United Information Systems Conference on Information Systems and eBusiness Technologies (UNISCON), volume 5 of $L N B I P$, pages $96-107$. Springer.

Gagné, D. and Trudel, A. (2009). TimeBPMN. In Proceedings of the IEEE Conference on Commerce and Enterprise Computing (CEC), pages 361-367. IEEE Computer Society. 
Godary, K. (2008). LPT : Little Parametric Tool, outil pour la validation d'une borne temporelle paramétrée. In CIFA'08: Conférence Internationale Francophone d'Automatique, Bucarest, Roumanie.

Guermouche, N. (2010). Etude des Interactions Temporisées dans la Composition de Services Web. $\mathrm{PhD}$ thesis, Université Henri Poincaré.

Guermouche, N. and Zilio, S. D. (2012). Towards Timed Requirement Verification for Service Choreographies. In 8th IEEE International Conference on Collaborative Computing: Networking, Applications and Worksharing, page 10, Pittsburgh, États-Unis.

Huai, W., Liu, X., and Sun, H. (2010). Towards Trustworthy Composite Service Through Business Process Model Verification. In IEEE, editor, Proceedings of the rth International Conference on Ubiquitous Intelligence $\&$ Computing and 7th International Conference on Autonomic $\&$ Trusted Computing (UIC/ATC), pages $422-427$.

Kallel, S., Charfi, A., Dinkelaker, T., Mezini, M., and Jmaiel, M. (2009). Specifying and Monitoring Temporal Properties in Web Services Compositions. In Eshuis, R., Grefen, P. W. P. J., and Papadopoulos, G. A., editors, Proceedings of the rth IEEE European Conference on Web Services (ECOWS), pages 148-157. IEEE Computer Society.

Kazhamiakin, R., Pandya, P. K., and Pistore, M. (2006a). Representation, Verification, and Computation of Timed Properties in Web. In Proceedings of the IEEE International Conference on Web Services (ICWS ), pages 497-504. IEEE Computer Society.

Kazhamiakin, R., Pandya, P. K., and Pistore, M. (2006b). Timed Modelling and Analysis in Web Service Compositions. In Proceedings of the 1st International Conference on Availability, Reliability and Security (ARES), pages 840-846. IEEE Computer Society.

Ling, S. and Schmidt, H. (2000). Time Petri nets for workflow modelling and analysis.
In Proceedings of the IEEE International Conference on Systems, Man, and Cybernetics, volume 4, pages 3039 -3044 vol.4. IEEE.

Makni, M., Hadj-Alouane, N. B., Tata, S., and Yeddes, M. M. (2011). Negotiating Deadline Constraints in Interorganizational Logistic Systems: A Healthcare Case Study. In Proceedings of the International Workshops and Education Track on Business Process Management Workshops (BPM), volume 100 of $L N B I P$, pages 108-118. Springer.

Makni, M., Tata, S., Yeddes, M. M., and Hadj-Alouane, N. B. (2010). Satisfaction and Coherence of Deadline Constraints in Inter-Organizational Workflows. In Proceedings of the Confederated International Conferences: CoopIS on On the Move to Meaningful Internet Systems: OTM 2010, volume 6426 of $L N C S$, pages 523-539. Springer.

Pesic, M., Schonenberg, M. H., Sidorova, N., and van der Aalst, W. M. P. (2007). Constraint-Based Workflow Models: Change Made Easy. In Proceedings of the Confederated International Conferences On the Move to Meaningful Internet Systems, volume 4803 of LNCS, pages 77-94. Springer.

Wang, F. (2004). Formal verification of timed systems: a survey and perspective . In Proceedings of the IEEE, pages 1283 1305.

Watahiki, K., Ishikawa, F., and Hiraishi, K. (2011). Formal Verification of Business Processes with Temporal and Resource constraints. In Proceedings of the IEEE International Conference on Systems, Man and Cybernetics, pages 11731180. IEEE.

Wong, P. Y. H. and Gibbons, J. (2009). A Relative Timed Semantics for BPMN. Electr. Notes Theor. Comput. Sci., $229(2): 59-75$. 